\title{
TERAPÊUTICA DE REPOSIÇÃO HORMONAL EM MULHERES MENOPAUSADAS: riscos e benefícios
}

\author{
ÉDISON APARECIDO ALVES \\ Formado em Farmácia e Bioquímica pela UNINOVE em julho de 2001
}

\author{
LUCIANA VISMARI \\ Farmacêutica-Bioquímica - UNESP; Mestre em Psicobiologia - UNIFESP; \\ Professora de Fisiologia da UNINOVE
}

\section{RESUMO}

Os distúrbios do climatério manifestam-se quando a produção e secreção de estrogênios começam a diminuir. São inúmeros os sintomas relacionados, sendo os principais a osteoporose, doenças cardiovasculares, alterações cutâneas e urinárias, sudorese, fogachos, ansiedade e irregularidades menstruais, os quais comprometem a qualidade de vida da mulher. Buscando uma melhoria dessas condições surgiu a Terapia de Reposição Hormonal - TRH, com á promessa de alívio dos sintomas e prevenção de uma série de doenças decorrentes dessa fase. Embora seu emprego venha aumentando na população de mulheres, os efeitos a médio e longo prazos são controversos. Nesse sentido, o objetivo deste trabalho foi avaliar a literatura a respeito dos riscos e benefícios da prescrição hormonal na menopausa. Numa análise cuidadosa dos trabalhos, verificou-se que as estimativas de riscos e benefícios diferem de acordo com as populações ou indivíduos estudados. A tendência atual é de que as tomadas de decisão fundamentem-se em evidências advindas dos estudos de ensaios clínicos randomizados. Uma análise individualizada dos riscos e benefícios também é indispensável, já que associada às possíveis vantagens há sempre uma série de prováveis desvantagens, que podem variar significativamente conforme o histórico de saúde e as características de cada paciente.

Palavras-chave: Terapia de Reposição Hormonal; menopausa; riscos; beneficios.

\section{ABSTRACT}

Climacteric disturbances occur when estrogen production and secretion start to reduce. There are a lot of symptoms related to this phase, as osteoporosis, cardiovascular diseases, coetaneous and genitourinary alterations, sudoresis, warmth, anxiety and menstrual irregularity, which implicate in impaired women life quality. Hormonal Replacement Therapy (HRT) arose searching an improvement of these conditions. HRT promises to reduce symptoms and to prevent many secondary diseases. In despite of an increase in its use among women populations, its effects at medium and long term are controversial. In this way, the objective of the present study was to evaluate the literature about risks and benefits of the hormonal prescription in the menopause. A careful analysis of several studies verified that risks and benefits differ among population or individuals studied. Actual tendency are decisions based in evidences from randomized clinical essays studies. An individualized analysis of risks and benefits is also indispensable, since possible advantages are also associated to probable disadvantages, which can vary significantly, depending on the historical health and characteristics of each patient.

Key words: Hormonal Replacement Therapy; menopause; risks; benefits. 


\section{INTRODUÇÃO}

\section{A MENOPAUSA E O ADVENTO DA TERAPÊUTICA DE REPOSIÇÃO HORMONAL -TRH}

A expectativa de vida da população elevou-se espetacularmente no século XX: no Império Romano era de 23 anos; à época do descobrimento da América, 27 anos; em 1850, era de 40 anos e, no início do século XX, atingiu 50 anos. Como a idade de entrada na menopausa permaneceu constante, uma porção crescente de mulheres vive um período cada vez mais longo de suas vidas no período pós-menopáusico (WISE et al., 1999). Durante os cerca de 30 anos dessa nova etapa da vida (climatério), quase inexistente até o século XX, a mulher é vítima da redução daqueles mesmos hormônios que a acompanharam durante anos (FERREIRA, 1999).

Os distúrbios do climatério manifestam-se quando a produção e a secreção dos estrogênios começam a diminuir. Mais de 100 sintomas são encontrados na menopausa, embora só os fenômenos vasoativos (fogachos) e a sudorese noturna estejam entre os quase universalmente encontrados e em clara dependência do hipoestrogenismo (BARRET-CONNOR; GRADE, 1998; SAMSIOE, 2001). Os primeiros sintomas observados são a irregularidade menstrual e/ ou amenorréia, os fogachos, sudorese, cefaléia, palpitação, tontura, fraqueza, insônia, irritabilidade, nervosismo, ansiedade, depressão, melancolia, esquecimento e dificuldade de concentração. Esses sintomas comprometem a qualidade de vida da mulher e são, na verdade, o marco inicial de um longo processo de alterações do metabolismo ósseo, dos lipídeos e de modificações da integridade funcional dos sistemas urogenital, psíquico e cutâneo. Como conseqüência da falta crônica de estrogênios, a osteoporose e as doenças cardiovasculares tendem a aparecer precocemente. Os problemas que ocorrem a médio prazo são principalmente os relacionados à atrofia, tais como: secura vaginal, dispareunia, infecção urinária, síndrome uretral, incontinência urinária de esforço sem causa anatômica e pele seca. A longo prazo ocorrem principalmente as doenças crônico-degenerativas como a osteoporose e sua maior conseqüência as fraturas; doenças cardiovasculares, como a hipertensão arterial sistêmica; doenças cardíacas isquêmicas, como o infarto agudo do miocárdio; acidente vascular cerebral e enfermidade de Alzheimer, ou demência senil (SAMSIOE, 2001).

O perfil sintomatológico da menopausa é heterogêneo. A variação da presença de fogachos e sudorese noturna, verdadeiros marcos deste período, é muito grande entre diferentes culturas, chegando a $70 \%$ nas norte-americanas, $76,6 \%$ nas latino-americanas e somente $18 \%$ nas chinesas (BLUMEL et al., 1992; GUTHRIE et al., 1996). Dieta, clima, genética e história reprodutiva contribuem para essas diferenças, que se tornam ainda mais evidentes quando sintomas como sexualidade, bem-estar e humor são considerados. (DEMETRIO, 2000).

Independentemente das diferenças sintomatológicas observadas, a idéia de que um aumento nos níveis sangüíneos de hormônios sexuais levaria a uma melhoria na qualidade de vida é bastante antiga, o que levou os antigos gregos e romanos, e mesmo os egípcios, a descreverem propriedades terapêuticas da alimentação com testículos de animais. No século XIX, a utilização de gônadas de animais ou seus extratos para o prolongamento do bem-estar e da juventude de homens e mulheres adquiriu popularidade, o que culminou, na década de 60 , no período de maior difusão da TRH (MELO et al., 1999). Coube a Sevringhaus (1929, apud Melo, 1999) comunicar o primeiro estudo norteamericano de sucesso sobre o uso desses preparados no tratamento dos sintomas da menopausa. A partir de então, houve rápido progresso e isolaram-se vários esteróides ovarianos, até que Dodds et al. (1938, apud Mello, 1999) sintetizaram o dietilestilbestrol, que se tornou o primeiro de uma série de compostos ativos por via oral com ação estrogênica, que viriam a ser utilizados na TRH. Assim, esta terapia consiste na realização de reposição hormonal com estrogênios sozinhos ou combinados à 
progesterona em mulheres na fase da menopausa (pré e pós).

Com a descoberta da utilização exógena de hormônios na TRH, vários estudos têm buscado formas de apresentação farmacêutica que permitam conforto, praticidade e segurança à paciente, a exemplo dos sistemas transdérmicos de liberação, géis, sistemas de liberação vaginal, implantes, sprays nasais e injeções (ANSEL et al., 2000; NETO et al., 2000). Além disso, diversos esquemas terapêuticos, tais como a administração de estrogênio cíclico ou contínuo, combinados ou não à progesterona cíclica ou contínua, têm sido utilizados na TRH, com diferentes vantagens e desvantagens (HEGG et al., 1995).

\section{RISCOS E BENEFÍCIOS DA TERAPIA DE REPOSIÇÃO HORMONAL (TRH)}

A discussão sobre as vantagens e desvantagens da TRH é tema de intenso debate no universo acadêmico e ganha repercussão na imprensa a partir dos posicionamentos relativamente dicotomizados dos pesquisadores aqueles que são 'a favor' propõem a manutenção de níveis adequados de estrógeno durante toda a vida da mulher, com base em seus comprovados efeitos profiláticos sobre a osteoporose e os transtornos cardiovasculares, independentemente de a mulher apresentar ou não sintomas e queixas relacionados ao hipoestrogenismo; os 'contrários' à prescrição indiscriminada da TRH a todas as menopausadas propõem seu uso para aquelas mulheres cujos sintomas não respondam a medidas gerais, como a alimentação rica em fitoestrógenos (caso da soja) e exercícios físicos, ou as que apresentem antecedentes de osteoporose ou doença instalada. Devem-se ressaltar os efeitos colaterais comprovados da terapia, como o câncer de endométrio (nos casos em que a TRH acontece sem oposição de progesterona ao estrógeno em mulheres não-histerectomizadas) e câncer de mama (BARRET-CONNOR; GRADE, 1998).

Um levantamento cuidadoso da literatura verificou que, no geral, a Terapia de Reposição Hormonal produz melhoras significativas na sintomatologia geral apresentada na menopausa (BREGIEIRO, 1999). No entanto, há vários aspectos que merecem ser avaliados e, para isso, segue um levantamento dos seus efeitos sobre os principais aspectos da saúde da mulher na menopausa:

- Osteoporose - pesquisas mostram que TRH aumenta a densidade mineral óssea, previne a perda óssea e reduz risco de osteoporose após a menopausa, sendo a melhor terapêutica no que se refere à relação custo-benefício (HALBE, 1999; NETO et al., 2000). Há evidências científicas demonstrando que, quando a paciente não está sob reposição estrogênica, a perda óssea pós-menopausa não é alterada pela ingestão de cálcio;

- Problemas cardiovasculares - apesar de vários estudos mostrarem índices menores de doenças coronárias em mulheres que fazem uso do estrogênio no pós-menopausa, seus efeitos cardioprotetores ainda não estão bem esclarecidos (BARRET-CONNOR; GRADE, 1998). Uma possível explicação pode basear-se em suas ações sobre o perfil lipídico, que levam à diminuição do colesterol e elevação da lipoproteína de alta densidade (HDL), além de possíveis efeitos sobre os vasos, com restauro da função endotelial, aumento da velocidade do fluxo arterial, diminuição da resistência vascular, da pressão arterial, da atividade fibrinogênica e de agregação plaquetária, e de uma provável ação antioxidante. A TRH não modifica o risco de acidente vascular encefálico, embora ele seja maior com a aplicação de doses elevadas de hormônio (NETO et al., 2000). O uso de progestagênios na prevenção de doenças coronarianas é controverso, pois alguns autores afirmaram que diminui o nível de HDL.

- Irregularidades menstruais, fogachos e sono - a TRH produz altos índices de promoção de regularidade menstrual e é considerada uma terapêutica eficiente para minimizar os sintomas vasomotores (fogachos) da menopausa, perturbadores do sono feminino nesta fase (BARRET-CONOR; GRADE, 1998). 
- Ansiedade e humor - um efeito positivo da Terapia de Reposição Hormonal estrogênica sobre o humor das mulheres climatéricas e no pós-menopausa está descrito na literatura, mas conclusões definitivas sobre este efeito elevador do humor do estrógeno são controversas devido às limitações metodológicas encontradas em muitos desses estudos (DEMETRIO, 2000).

- Doença de Alzheimer - aqui os resultados são contraditórios: pequeno número de estudos sugere que a TRH pode proteger contra o desenvolvimento da demência. (TANG et al., 1996).

- Sistema urogenital - a atrofia da mucosa urogenital é um fator que pode contribuir para a diminuição da satisfação sexual e uma indicação importante para a terapêutica estrogênica (HURD, 1996). Com a administração de estrogênios, o número destes receptores na uretra, vagina e bexiga retornam aos níveis pré-menopausa, o que melhora a perda urinária em $50 \%$ das mulheres, por aumento da espessura da mucosa uretral (ROSSI et al., 2001).

- Risco de câncer endometrial - o uso de estrogênios isolados pela mulher climatérica leva a um aumento no risco de desenvolver hiperplasia endometrial e carcinoma de endométrio, a depender da dose e duração da administração. Com uma dose normal, o aumento é de 4 a 5 vezes maior quando o tratamento com estrogênios se prolonga por mais de quatro ou cinco anos (SAMSIOE, 2001). Muitos autores defendem a idéia de que, nas mulheres com útero, existe a necessidade de associar um progestagênio, de forma que o risco para desenvolver essas lesões passe a ser o mesmo ou até menor do que o apresentado pelas mulheres em menopausa que não utilizam a TRH (MELO et al., 1999; LISBOA; WANNACHER, 2001). Pacientes histerectomizadas dispensam o emprego de progestagênios, cuja função primordial em TRH recai sobre a prevenção dos estados hiperplásicos do endométrio (FERNANDES et al., 2000).
- Risco de câncer de mama - existe muita controvérsia no que se refere ao risco de câncer de mama devido à TRH. Várias metanálises sugeriram desde a ausência de risco aumentado até um alto risco. Recente análise de 51 estudos epidemiológicos (incluindo cerca de $90 \%$ dos dados mundiais), entretanto, revelou um aumento de 2,3\% no risco relativo de câncer de mama para cada ano de uso da TRH. E mais importante que a dose parece ser a duração do tratamento, havendo um risco maior após 5-10 anos de uso. O uso conjunto de progestagênios não diminui, indicando até mesmo aumento de tal risco; em contrapartida, há evidências de que a mortalidade relacionada ao câncer de mama pode ser menor em pacientes submetidas a estrogenioterapia (NETO et al., 2000; LISBOA; WANNACHER, 2001). Assim, o câncer de mama não-tratado, antecedentes pessoais de hiperplasia atípica de mama, câncer de mama aparentemente curado e histórico familiar de câncer de mama constituem contra-indicações para a TRH (LISBOA; WANNACHER, 2001).

\section{CONSIDERAÇÕES FINAIS}

A análise da literatura permitiu concluir, de um lado, que a TRH possui uma série de vantagens e, de outro, que é necessário considerar cuidadosamente cada uma das desvantagens que apresenta. Quando o clínico planeja uma TRH, todo o esforço deve ser feito para selecionar a forma de tratamento clinicamente mais apropriada e preferida para cada paciente, visando melhoras na sua qualidade de vida. As mulheres têm históricos diferentes de saúde e de responsividade ao tratamento, o que resulta em necessidades diferentes de TRH. Assim, cumpre atentar para o fato de que a reposição hormonal, quando realizada, deve sempre ser individualizada, não sendo possível a padronização do tipo de tratamento utilizado.

Além disso, o profissional farmacêutico, dentro de uma equipe multidisciplinar, precisa estar atento aos diferentes tipos de tratamento, auxiliando o clínico na busca de alternativas para 
minimizar o desconforto presente no período de menopausa. Nesse sentido, para os pacientes que não podem ser submetidas à $\mathrm{TRH}$, existem tratamentos alternativos disponíveis no mercado, tais como: moduladores seletivos de receptores de estrogênio (NETO et al., 2000), fitoestrógenos (URBANETZ et al., 2001) e tibolona (NAHAS et al., 2001), sobre os quais a literatura ainda prescinde de estudos capazes de esclarecer dúvidas restantes acerca da eficácia no tratamento e prevenção dos sintomas relacionados à menopausa.

\section{REFERÊNCIAS BIBLIOGRÁFICAS}

ANSEL, H.C; POPOVICH, N. G.; ALLEN, L. V. Farmacotécnica: formas farmacêuticas; sistemas de liberação de fármacos. 6. ed. São Paulo: Premier, 2000. 569 p.

BARRETT-CONNOR, E. Y.; GRADY, D. Hormone replacement therapy, heart disease and other considerations. Ann. Rev. Public. Health, 19, p. 5572, 1998.

BLUMEL, J.E.; BRANDT, A.; TACLA, X. Perfil sintomático de la mujer climatérica: experiencía clínica. Rev. Med. Chile, 120(9), p. 1017-1021, 1992.

BREGIEIRO, L.O. Adesão à terapêutica de reposição hormonal. Reprodução; Climatério, 14 (3), p. 141-144, 1999.

DEMETRIO, F.N. Efeito da terapia de reposição estrogênica sobre o humor e a ansiedade em mulheres menopausadas. São Paulo, 2000, 202p. Tese apresentada à Faculdade de Medicina da Universidade de São Paulo para obtenção do título de Doutor em Medicina.

FERNANDES, C.E. et al. Efeitos da associação trifásica 17-beta-estradiol/acetato de noretisterona no controle das irregularidades menstruais em pacientes na perimenopausa. Revista Brasileira de Medicina, S57, p. 30-37, 2000.

FERREIRA, J.A.S. A perimenopausa. In: FERNANDES, C.E. et al. Climatério feminino: fisiopatologia, diagnóstico e tratamento. São Paulo: Lemos, 1999. p. 41-56.

GUTHRIE, J.R.; DENNERSTEIN, L.; HOPPER, J.L.; BURGER, H.G. Hot flushes, menstrual status and hormone levels in a population-based sample of midlife women. Obstet. Gynecol., 88(3), p. 437-442, 1996.
HALBE, H.W. Osteoporose pós-menopausal: como diagnosticar e tratar. Revista Brasileira de Medicina, S56, p. 235-245, 1999.

HEGG, R. Vias e esquemas de administração de hormônios esteróides. In: PINOTTI, J.A. Menopausa. São Paulo: Roca, 1995. p. 227-233.

HURD, W.W. Menopausa. In: NOVAK, J. Tratado de Ginecologia. 12. ed. São Paulo: Atheneu, 1996. p. 696-712.

LISBOA, K.O.; WANNACHER, L. Reposição Hormonal na menopausa: benefícios e riscos. Reprodução e Climatério, 16 (1), p. 11-19, 2001.

MELO, N. R. Efeito da terapêutica estrogênica transdérmica associada ao progestagênio oral sobre o perfil lipídico e a remoção plasmática de uma emulsão rica em colesterol. São Paulo, 1999, 74p. Tese apresentada à Faculdade de Medicina da Universidade de São Paulo para obtenção do título de Professor Livre-Docente, junto ao Departamento de Obstetrícia e Ginecologia.

NAHAS, E.A.P.; PONTES, A.; NAHAS NETO, J.; TRAIMAN, P.; DE LUCA , L; ABBADE, J. Efeitos da atividade física e da tibolona sobre a densidade mineral óssea em mulheres na pós-menopausa. Reprodução; Climatério, 16 (1), p. 47-52, 2001.

NETO, J.S.P. et al. Climatério - abordagem terapêutica. In: VILAR, L. et al. Endocrinologia Clínica. 2. ed. Rio de Janeiro: Medsi, 2000. p. 496-508.

ROSSI, P. et al. Distúrbios urogenitais no climatério. Revista Brasileira de Medicina, 58 (6), p. 370-380. 2001.

SAMSIOE, G. Menopausa e terapia de reposição hormonal. 2. ed. São Paulo: Merit Publishing International, 2001.144p.

TANG, M.X.; JACOBS, D.; STERN, Y.; MARDER, K.; SCHOFIELD, P.; GURLAND, B.; ANDREWS, H.; MAVEUX, R. Effect of estrogen during menopause on risk and age at onset of Alzheimer's disease. Lancet, 348 (9025), p. 429-432. 1996.

URBANETZ, A. A.; RIBEIRO, M. C. Fitoestrogênicos: verdade ou mito? Reprodução; Climatério, 16 (2), p. 92-97. 2001.

WISE, P.M.; SMITH, M.J.; DUBAL, D.B.; WILSON, M.E.; KRAINAK, K.M.; ROSEWELL, K.L. Neuroendocrine influences and repercussions of the menopause. Endocr. Rev., 20 (3), p. 243-248. 1999. 


$$
\longrightarrow
$$

